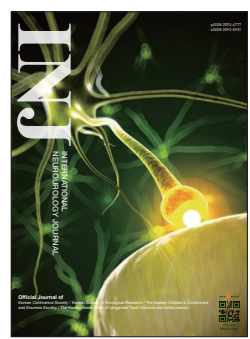

\title{
Treatment of Stress Urinary Incontinence in Neurological Patients With an Injectable Elastomer Prosthesis: Preliminary Results
}

\author{
Julien Renard ${ }^{1,2}$, Marco Citeri ${ }^{3}$, Lucia Zanollo ${ }^{3}$, Chiara Guerrer ${ }^{3}$, Luigi Rizzato ${ }^{3}$, Luca Frediani ${ }^{3}$, Christophe Iselin ${ }^{1}$, Michele Spinelli ${ }^{3}$ \\ ${ }^{1}$ Divison of Urology, Geneva University Hospital, Geneva, Switzerland \\ ${ }^{2}$ Division of Neuro-Urology, Ospedale Regionale di Bellinzona and Neurocentro della Svizzera Italiana, Lugano, Switzerland \\ ${ }^{3}$ Spinal Unit, Division of Neurourology, A. Zanollo Center for Sacral Area Disfunction, Niguarda Hospital, Milan, Italy
}

Many treatment options for stress urinary incontinence are difficult to apply to neurological patients. Urolastic is a new agent that is primarily indicated for women with mild stress urinary incontinence or men after prostate surgery. In this report, we present a series of 5 cases describing the first use of Urolastic to treat neurological patients. All patients were evaluated with a voiding diary and the use of auxiliary devices as the main indicators of continence. The median operative time was 30.8 minutes, and no complications were observed. Of the 5 patients, 4 reported improved incontinence: 2 switched from diapers to small pads, while the other 2 patients were able to discontinue urinary condom use. The only instance of treatment failure occurred in a patient with a low-compliance bladder. The advantages of this procedure appear to include a soft-cuff effect, reversibility, and minimal invasiveness. However, a future randomized study would be necessary to validate this treatment option.

Keywords: Urinary Incontinence, Stress; Urinary Bladder, Neurogenic; Injectable; Bulking

- Conflict of Interest: No potential conflict of interest relevant to this article was reported.

\section{INTRODUCTION}

Stress urinary incontinence (SUI) is common in neurological patients with subsacral lesions (spinal cord lesions, spina bifida, or myelomeningocele). These patients can experience underactive bladder and stress incontinence due to sphincter insufficiency. Unfortunately, the management of SUI has always been more challenging than the management of urge incontinence [1]. Various treatment options exist, such as artificial sphincters and hydraulic prostheses, which are considered effective but expose patients to an elevated risk of erosion [2]. Mtany injectable agents have already been described. Unfortunately, they often provide insufficient bulking and are likely to undergo migration [3], as well as being associated with possible inflamma- tory reactions and being difficult to remove.

Nonetheless, new products have been designed with primary indications for women with mild SUI or men after prostate surgery. Urolastic (Urogyn BV, Nijmegen, The Netherlands) is a new bulking agent, the efficacy of which has already been described in the management of female SUI [4], and is considered to be an effective and long-lasting treatment, with more than 1 year of efficacy. It is composed of a titanium-coated, nonbioabsorbable vinyl dimethyl polydimethylsiloxane elastomer. It can considered to be a hybrid between a bulking agent, as it is injected at the midurethra, and a prosthesis, as it creates a soft-cuff effect by solidifying around the urethra, preventing migration (according to the manufacturer). So far, its use in the treatment of neurological patients has not been described, which is the aim of this case series.

Corresponding author: Julien Renard (iD http://orcid.org/0000-0003-3390-1502 Division of Urology, Geneva University Hospital, 4 rue Gabrielle Perret-Gentil 1211, Geneva, Switzerland

E-mail: Julien.renard@hcuge.ch / Tel: +41223727963 / Fax: +41223721965

Submitted: May 20, 2016 / Accepted after revision: July 19, 2016 


\section{CASE REPORT}

Treatment was indicated in patients with symptomatic SUI due to intrinsic sphincter insufficiency linked exclusively to a lowercord lesion. Patients with organ prolapse, a history of previous radiotherapy, or who had undergone a procedure in the perineum or urethral region were not considered for this treatment.

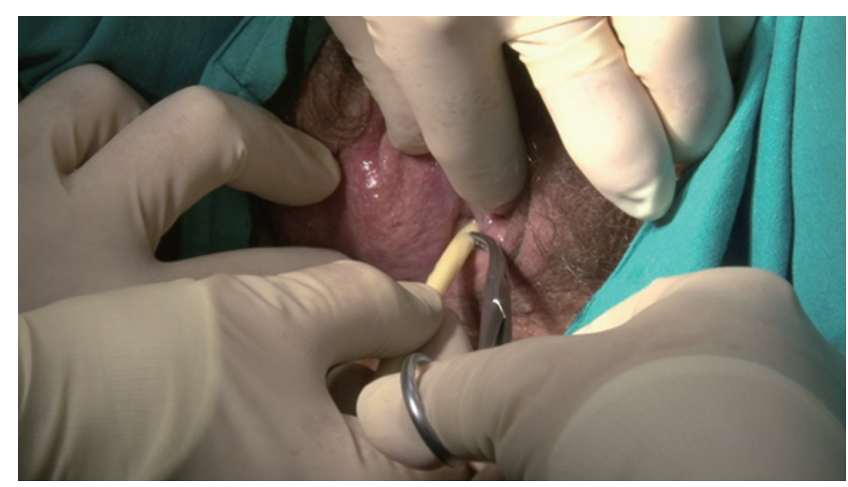

Fig. 1. Measurement of urethra length using a foley catheter.

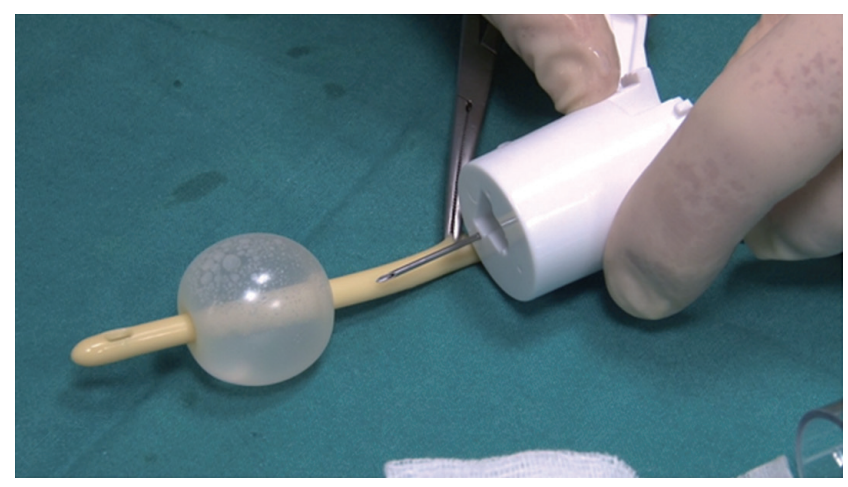

Fig. 2. Positioning of applicator in order to perform injections at mid urethra.

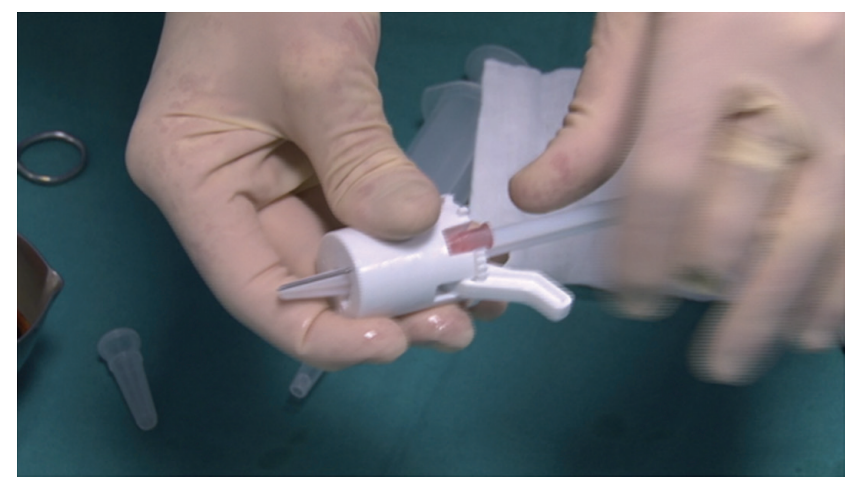

Fig. 3. View of applicator positioned for mid urethra injection.
Five patients were identified. All subjects were offered the possibility of treatment with this new agent or using other devices. They were informed of its novel characteristics and provided written informed consent for the procedure. The patients' grade of incontinence was assessed preoperatively based on their need for and number of auxiliary devices (condoms or diapers). Postoperative continence was defined as perfect when dryness

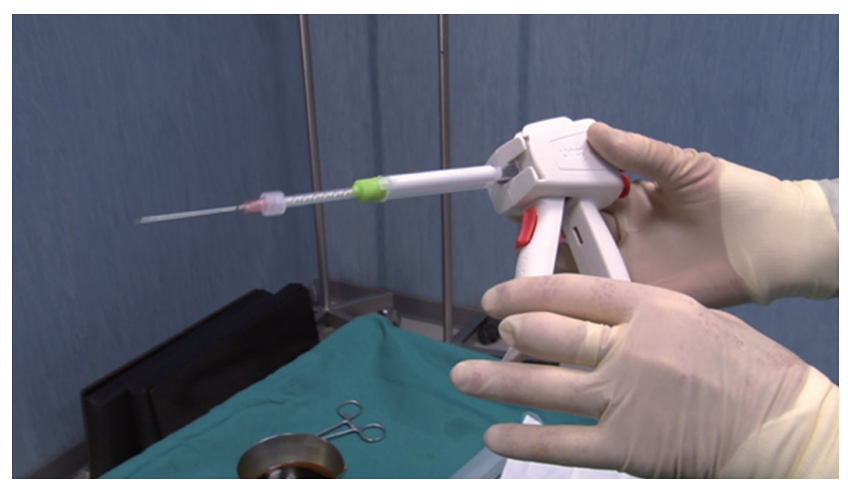

Fig. 4. Injection "gun" with mounted syringe.

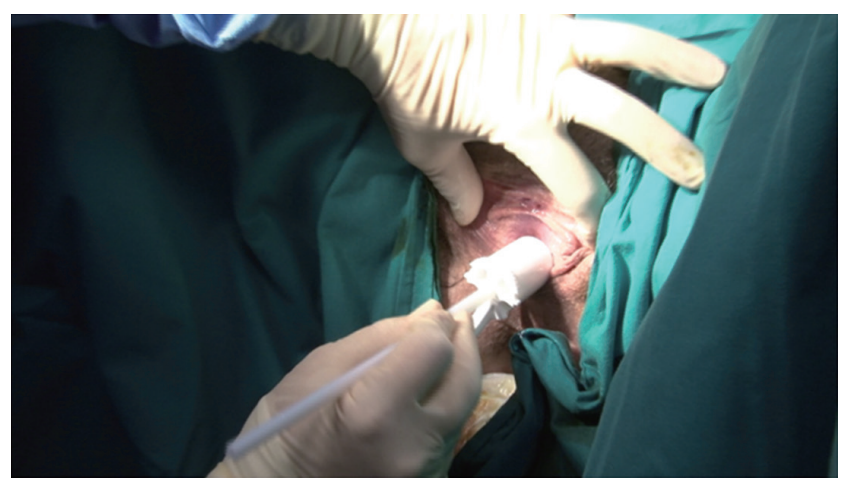

Fig. 5. Applicator after insertion in the urethra.

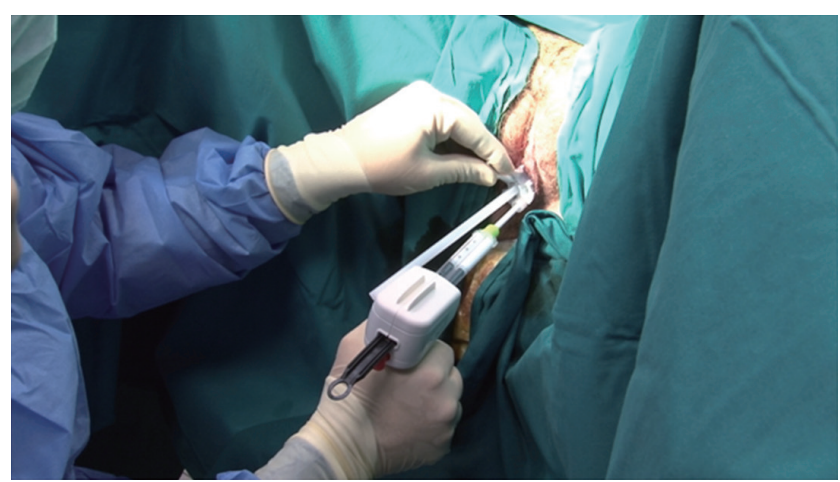

Fig. 6. Injection of urolastic through applicator. 
occurred between regular micturition or self-catheterization. Persistent incontinence was defined as occurring when a patient did not observe any improvement or changes in device use, whereas the treatment of incontinence was considered successful when the patient observed significant improvements, and reduced the use of auxiliary devices or changed the type of pads.

The procedure was performed under local anesthesia with the patient in the dorsal lithotomic position. Urolastic (Urogyn, Nijmegen, The Netherlands) was obtained in a prefilled, sterile, dual container of $5 \mathrm{~mL}$ ( 2 syringes $\times 2.5 \mathrm{~mL}$ ), supplied with a static mixer that allows for adequate premixing of the syringe content. In women, the procedure was performed without a cystoscope. The bladder was filled with $200 \mathrm{~mL}$ of saline solution, and using a simple Foley catheter which was put in tension, the length of the urethra was measured and marked on the catheter (Figs. 1-3). Before the injection, the patient was asked to cough in order to evaluate grade of urine loss This length was recorded on the applicator in order to perform the injection with an 18-gauge needle at the midurethra (Figs. 4-6)

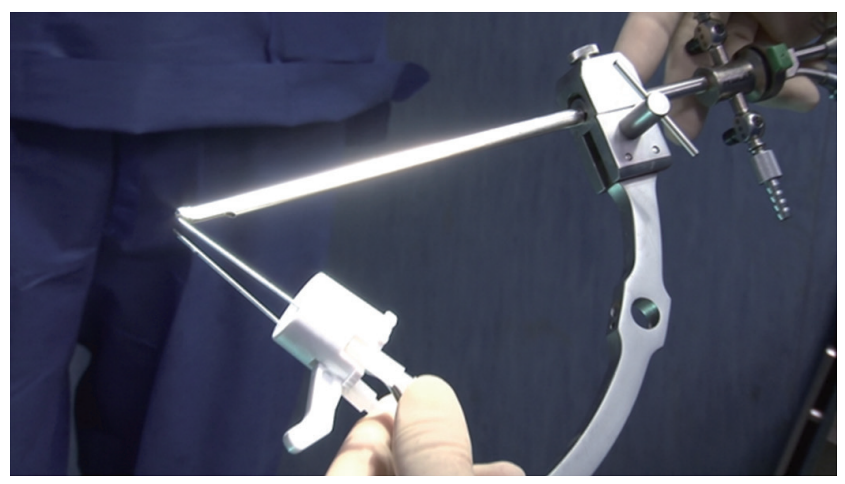

Fig. 7. "Sextant" mounted on cystoscope for perineal injection in male patients.

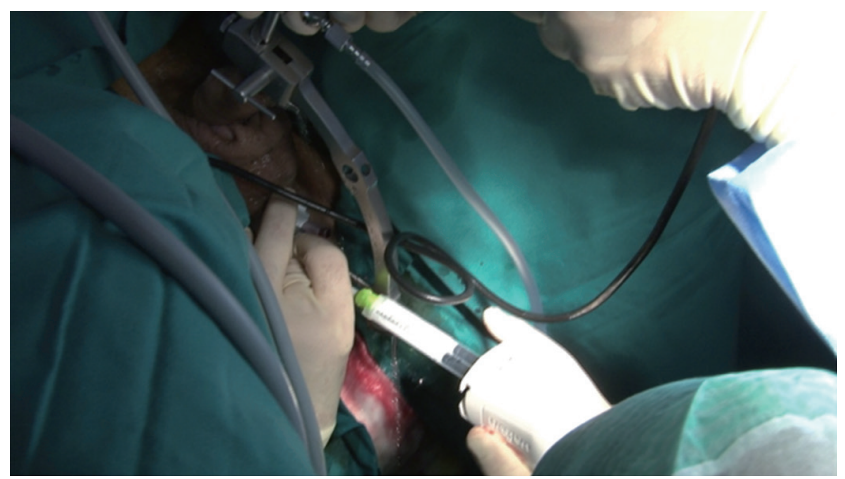

Fig. 8. Perineal injection in male patients. at 4 sites (12 o'clock, 3 o'clock, 6 o'clock, and 10 óclock). In male patients, the injection was performed at 5 oclock and 9 o'clock, in the membranous midportion of the male urethra, under direct vision thanks to a dedicated device placed on the cystoscope (Figs. 7-10). At the end of the procedure, the bladder was filled with $200 \mathrm{~mL}$ of saline and the cough test was performed. Outcomes were assessed in examinations performed at regular intervals ( 1,6 , and 12 months), the results of which are summarized in Table 1 . Of the 5 patients, 2 were male and 3 were female. Their mean age was 48 years (range, $40-65$ years). Two

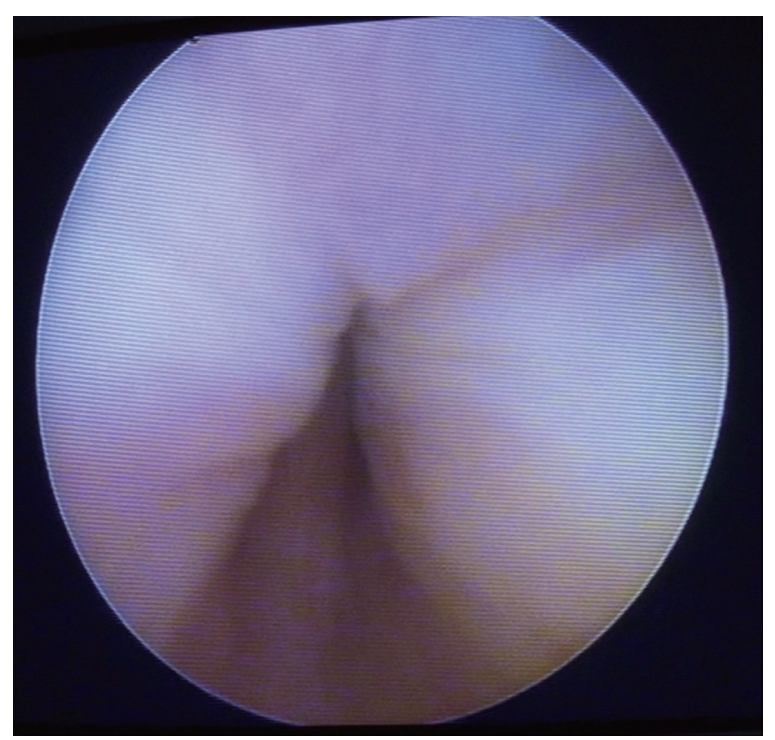

Fig. 9. Endoscopic view of membranous urethra before injection.

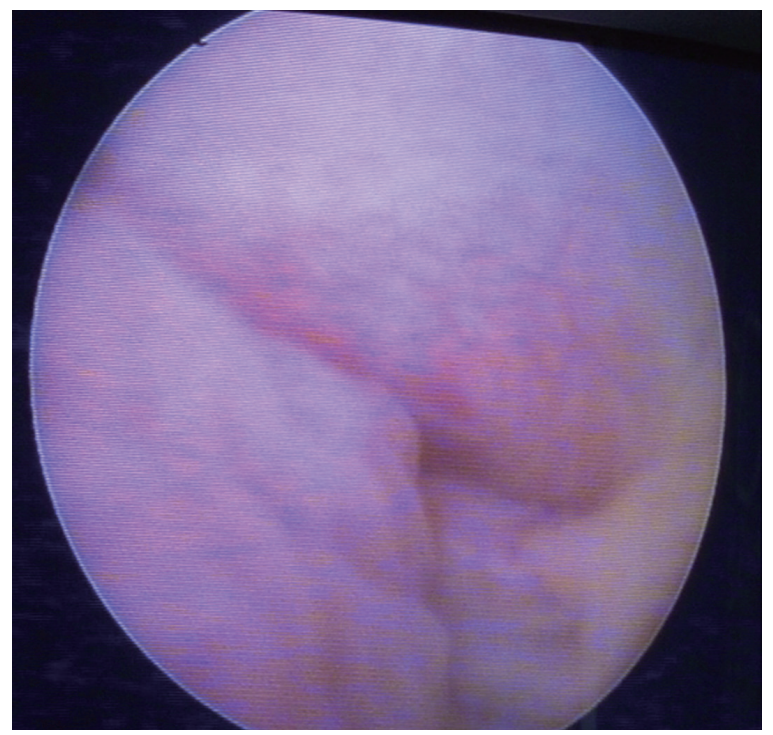

Fig. 10. Endoscopic view of membranous urethra after injection. 
Table 1. Pre- and postoperative results

\begin{tabular}{lcllll}
\hline Sex & Age $(\mathrm{yr})$ & Neurogenic disorder & Incontinence grade & Pretreament & Posttreatment \\
\hline Female & 65 & Medullary ischemia & Complete & Diaper & Small pad (1/day) \\
Female & 42 & Spina-Bifida & $50 \%$ of urine loss & Diaper & Small pad (1/day) \\
Female & 50 & Myelitis & Complete & Diaper & No progress \\
Male & 41 & Spine lesion L1 ASIA C & $50 \%$ of urine loss & Condom & Dry \\
Male & 51 & Medullary ischemia & $50 \%$ of urine loss & Condom & Dry (self-catheterization up to 500 mL)
\end{tabular}

ASIA, American Spinal Injury Association.

patients had medullary ischemia, and there was 1 case of myelomeningocele, 1 case of infectious myelitis and 1 case of an incomplete spinal cord (L1 American Spinal Injury Association C). The grade of leakage was based on subjective symptoms and pad testing (number and weight of pads), with full incontinence in 2 cases and loss of 50\% of bladder capacity in the remaining patients, as assessed through urodynamic testing. Preoperatively, all patients required either a urinary condom or diaper (mean, 3 per day). The mean operative time was 45 minutes. No complications were recorded perioperatively or postoperatively.

Within a mean follow-up period of 7.8 months (range, 5-12 months) after surgery, 4 of the 5 patients reported subjective improvements. In the male patients, the objective results, as assessed by a bladder diary, showed complete resolution with the discontinuation of urinary condom use. In 1 male patient, underactive bladder was associated with sphincter insufficiency. In the postoperative assessments, that patient reported selfcatheterization for volumes up to $500 \mathrm{~mL}$, with complete dryness between catheterizations. The other male patient reported being completely dry, without even the use of pads. Two of the female patients presented improvements, shifting from diapers (mean use of 2-3 thoroughly wet diapers per day) to the use of 1 small pad. The only treatment failure was observed in a female patient with a bladder that showed low compliance in a preoperative urodynamic assessment.

\section{DISCUSSION}

The treatment of SUI remains challenging in nonneurological patients. The addition of a neurogenic condition makes management even more difficult. We present findings that demonstrate the efficacy of a novel injectable agent in the specific setting of neurological patients. In these 5 cases, all but 1 patient benefitted from the procedure. The only treatment failure was observed in a patient with a low-compliance bladder $(<30 \mathrm{~mL} /$ $\mathrm{cm} \mathrm{H}_{2} \mathrm{O}$ ). This was due to the rapid rise of bladder pressure, exceeding the pressure applied to the urethra by the injected agent, resulting in urine loss before reaching an acceptable selfcatheterization volume. Based on our experience, it is difficult to estimate the minimum requirement for bladder compliance, as the number of patients is too limited, but this aspect of treatment should be investigated in a future randomized trial in order to fully define the correct indications for this device. Nonetheless, preoperative urodynamic assessments appear to be compulsory.

This procedure appears to be a possible valid alternative to other available techniques. Although the gold-standard treatment remains artificial sphincter placement, artificial sphincters have potential complications in neurological patients, including the frequent need for intermittent self-catheterization, increasing the incidence of cuff erosion; the pressure applied to the bulbar urethra in wheelchair-bound patients; the open bladder neck that occurs with sacral cord lesions, with the urine-filled prostatic urethra acting as a potential source of infection when a bulbar cuff is placed; and risks linked to endoscopic treatment that may contribute to the erosion of bulbar cuffs [5]. The soft-cuff effect is potentially advantageous in these cases, as in the long term it might reduce the risk of erosion compared to artificial sphincters or Adjustable Continence Therapy (Medtronic, MN, Minnesota, USA) balloons, which have a more rigid composition. Urolastic hardens in situ into a flexible rubberlike plug, which adapts itself to the shape of the local environment [4]. We acknowledge that this advantage is only hypothetical for the moment, and should be confirmed by cystoscopy; nevertheless, none of the patients presented with clinical suspicion or signs of urethral erosion. We believe that this characteristic of Urolastic might be confirmed by a larger randomized study.

Its composition may also be an advantage in contrast to oth- 
er agents that pose a risk of migration [6,7]. Many promising agents have received preliminary approval from the U.S. Food and Drug Administration, before being subsequently withdrawn for this reason, such as autologous fat and Teflon [8]. In our experience, patients did not complain of the loss of efficacy, confirming the observations made by Zajda and Farag [4], who did not encounter migration in 19 patients over a 12-month follow-up period. He stated that Urolastic was effective and durable, which can be explained by the flexibility of the implant, which enables it to adapt itself to the shape of the local environment during injection, reducing the likelihood of migration. Zajda and Farag [4] also stated in the same article that Urolastic is a biocompatible and nonbiodegradable agent, contributing to its long-term efficacy. In the present case series, we were able to confirm these findings in neurological patients. Nonetheless, it is important to acknowledge that our conclusions were only based on patient satisfaction and that, in order to conclusively demonstrate that this agent poses a low risk of migration, a structured study should be conducted, including a specific search for migration, most likely using imaging due to the radio-opacity of Urolastic.

Its minimal invasiveness and reversibility are other advantages of Urolastic. Injection is fast, easy, and requires at most the introduction of a cystoscope, in contrast to classical prosthesis procedures, which can be performed either via open or robotic surgery [5], leading to problems with costs. Furthermore, solidification of the agent and its radio-opacity may make it easily retrievable in the event of the complications reported by other authors [3], such as abscess formation, bladder hyperactivity, and dyspareunia, although these complications have yet to be reported for Urolastic.

Urolastic therefore appears to be a feasible, safe, and durable treatment option for SUI in neurological patients based on this series. It is important to state that this case series is small, and as is true for all bulking agents, our findings should be confirmed in a randomized controlled trial in a homogeneous neurological patient population with a long period of follow-up in order to draw valid conclusions. However, we believe that this case series is important, as it represents the first step in evaluating a new device for the management of a condition with a limited range of treatment options.

In conclusion, these preliminary results show that this procedure is worthy of a larger randomized study, because, although it was performed in only a few cases, the technique appears to be feasible, safe, and effective in neurological patients. We believe that its potential advantages in comparison with other treatment options are: (1) the creation of a soft-cuff effect, as it is easily and completely removable, (2) the absence of a risk of migration, and (3) the fact that it is minimally invasive.

Further studies with larger patient samples will provide more valid insights into this new treatment option.

\section{REFERENCES}

1. Groen J, Pannek J, Castro Diaz D, Del Popolo G, Gross T, Hamid R, et al. Summary of European Association of Urology (EAU) Guidelines on Neuro-Urology. Eur Urol 2016;69:324-33.

2. Drake WJ, Apostolidis A, Emmanuel A, Gajewski J, Harrison SC, Heesakkers J, et al. Neurologic urinary and faecal incontinence. In: Abrams P, Cardozo L, Khoury S, Wein A. Incontinence. 5th ed. Bristol (UK): International Consultation on Urological Diseases and European Association of Urology; 2013. p. 827-1000.

3. Davis NF, Kheradmand F, Creagh T. Injectable biomaterials for the treatment of stress urinary incontinence: their potential and pitfalls as urethral bulking agents. Int Urogynecol J 2013;24:913-9.

4. Zajda J, Farag F. Urolastic-a new bulking agent for the treatment of women with stress urinary incontinence: outcome of 12 months follow up. Adv Urol 2013;2013:724082.

5. Yates DR, Phé V, Rouprêt M, Vaessen C, Parra J, Mozer P, et al. Robot-assisted laparoscopic artificial urinary sphincter insertion in men with neurogenic stress urinary incontinence. BJU Int 2013; 111:1175-9.

6. Pannek J, Brands FH, Senge T. Particle migration after transurethral injection of carbon coated beads for stress urinary incontinence. J Urol 2001;166:1350-3.

7. Sandhu JS. Treatment options for male stress urinary incontinence. Nat Rev Urol 2010;7:222-8.

8. Zoorob D, Karram M. Bulking agents: a urogynecology perspective. Urol Clin North Am 2012;39:273-7. 